\title{
Integrated avionics architectures
}

Reinhard Reichel

ILS, Universität Stuttgart

This manuscript is not available according to publishing restriction. Thank you for your understanding. 